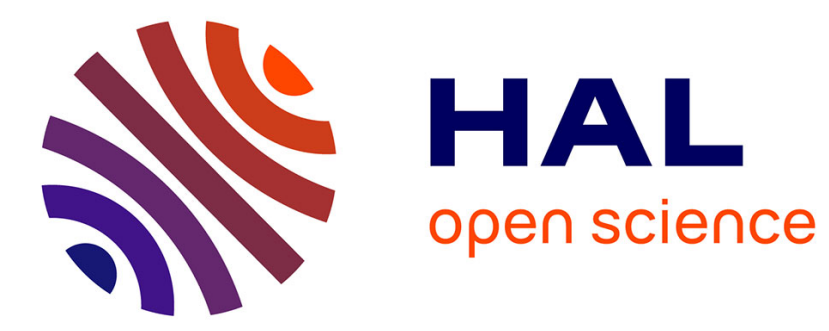

\title{
New results in guided waves in heterogeneous elastic media
}

\author{
Patrick Joly, Ricardo Weder
}

\section{To cite this version:}

Patrick Joly, Ricardo Weder. New results in guided waves in heterogeneous elastic media. [Research Report] RR-1377, INRIA. 1991. inria-00075184

\section{HAL Id: inria-00075184 https://hal.inria.fr/inria-00075184}

Submitted on 24 May 2006

HAL is a multi-disciplinary open access archive for the deposit and dissemination of scientific research documents, whether they are published or not. The documents may come from teaching and research institutions in France or abroad, or from public or private research centers.
L'archive ouverte pluridisciplinaire HAL, est destinée au dépôt et à la diffusion de documents scientifiques de niveau recherche, publiés ou non, émanant des établissements d'enseignement et de recherche français ou étrangers, des laboratoires publics ou privés. 


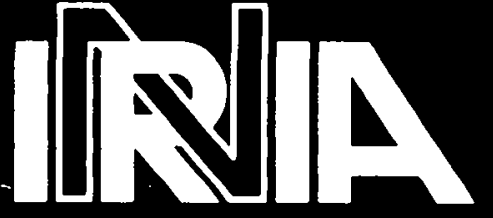

UNITÉ DE RECHERCHE URIA-ROCOUENCOURT

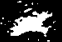

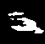

Institut National de Recherche en Informatique et en Automatique

Domaine de Voluceau - Rocquencourt

$$
\text { B.P.105 }
$$

78153 Le Chesnay Cedex

$$
\text { France }
$$

Tél.:(1)39635511

\section{Rapports de Recherche}

$$
N^{\circ} 1377
$$

\section{Programme 6}

Calcul scientifique, Modélisation et Logiciels numériques

\section{NEW RESULTS IN GUIDED WAVES IN HETEROGENEOUS ELASTIC MEDIA}

Patrick JOLY

Ricardo WEDER 


\section{NEW RESULTS IN GUIDED WAVES IN HETEROGENEOUS \\ ELASTIC MEDIA}

\section{NOUVEAUX RESULTATS SUR LES ONDES GUIDEES EN MILIEU ELASTIQUE HETEROGENE}

Patrick Joly (1)

Ricardo Weder $(2)(+)$

\section{Abstract}

We prove the existence of guided waves propagating with velocity strictly larger than the $S$ wave velocity at infinity in the case of unbounded elastic media invariant under translation in one space direction and asymptotically homogeneous at infinity.

These waves correspond to the existence of eigenvalues embedded in the essential spectrum of the selfadjoint elastic propagator.

\section{Résumé}

Nous démontrons l'existence d'ondes guidées se propageant à une vitesse strictement supérieure à la vitesse des ondes $S$ à l'infini dans le cas d'un milieu élastique hétérogène infini, invariant par translation dans une direction d'espace et asymptotiquement homogène à l'infini.

Ces ondes correspondent à l'existunce de valeurs propres plongées dans le spectre essentiel de l'opérateur auto-adjoint associé au problème.

\section{$\underline{\text { Key Words }}$}

Elastic waves, guided waves, spectral theory, eigenvalues embedded in the essential spectrum.

\section{Mots Clés}

Ondes élastiques, ondes guidées, théorie spectrale, valeurs propres plongées dans le spectre essentiel.

(1) inria. Domaine de Voluceau 78153 Le Chesnay Cédex. France

$\left({ }^{2}\right)$ Instituto de Investigaciones en Matemáticas Aplicsdas y en Sistemas. Universidad Nacional Autónoma de México. Apartado Postal 20-726. México D.F. 01000.

$\left({ }^{\dagger}\right)$ Fellow. Sistema Nacional de Investigadores. 


\section{Introduction}

In this paper we study the propagation of guided waves in an isotropic elastic medium which is invariant in one space direction. Let us denote by $\left(x, x_{3}\right), x=$ $\left(x_{1}, x_{2}\right) \in \mathbb{R}^{2}, \quad x_{3} \in \mathbb{R}$, the generic point in $\mathbb{R}^{3}$, and by $t \in \mathbb{R}$, time. By $U\left(x, x_{3}, t\right)=$ $\left(U_{1}\left(x, x_{3}, t\right), U_{2}\left(x, x_{3}, t\right), U_{3}\left(x, x_{3}, t\right)\right)$ the displacement field, where $U_{i}\left(x, x_{3}, t\right)$ are func-

tions from $\mathbb{R}^{4}$ into $\mathbb{R}$. The equations of elastodynamics governing the propagation of waves in such a medium are written as $[\mathbf{A}]$

$$
\rho(x) \frac{\partial^{2} U_{i}}{\partial t^{2}}-\sum_{j=1}^{3} \frac{\partial}{\partial x_{j}} \sigma_{i j}(U)=0
$$

for $i=1,2,3$.

$$
\begin{gathered}
\sigma_{i j}(U)=\lambda(x) \operatorname{div} U \delta_{i j}+2 \mu(x) \epsilon_{i j}(U), \\
\operatorname{div}(U)=\sum_{i=1}^{3} \frac{\partial}{\partial x_{i}} U_{i}\left(x, x_{3}, t\right), \\
\epsilon_{i j}(U)=\frac{1}{2}\left(\frac{\partial}{\partial x_{j}} U_{i}\left(x, x_{3}, t\right)+\frac{\partial}{\partial x_{i}} U_{j}\left(x, x_{3}, t\right)\right),
\end{gathered}
$$

$i, j=1,2,3$.

The invariance of the medium under translations in the direction $x_{3}$ appears in the fact that the density $\rho(x)$ and the Lamē coefficients $\lambda(x)$ and $\mu(x)$ are independent of the coordinate $x_{3}$. In this paper we will furthermore assume that $\rho$ and $\mu$ are positive 
and constant as functions of $x$, and that $\lambda(x)$ is a bounded above and below measurable function of $x$. We denote

$$
\begin{gathered}
\lambda_{-}=\text {ess inf } \lambda(x)>0, \\
\lambda_{+}=\text {ess } \sup \lambda(x)<\infty .
\end{gathered}
$$

Assume that

$$
\left|\lambda(x)-\lambda_{\infty}\right| \leq C(1+|x|)^{-\delta}
$$

for some positive constants $\lambda_{\infty}, C, \delta$.

A guided wave (or guided mode) is by definition a particular solution of equations (1.1) which has the following form

$$
U\left(x, x_{3}, t\right)=\tilde{u}(x) e^{i\left(\omega t-\beta x_{3}\right)}
$$

where $\omega>0, \beta>0$, and the amplitude field $\tilde{u}(x)$ is square integrable with respect to $x:$

$$
\int|\tilde{u}(x)|^{2} d x<\infty
$$

The wave defined by (1.8) is harmonic (we take the usual convention of allowing for complex valued amplitudes). It propagates in the $x_{3}$ direction with phase velocity 
$\omega / \beta$. Its energy remains localized in the transverse directions, $x$, because of (1.9). As in classical situations such waves can exist only if the wave number $\beta$ and the pulsation $\omega$ are related by the dispersion relation of the mode.

Following [BDJ] we formulate this problem as an eigenvalues and eigenvectors problem for a family of selfadjoint operators in Hilbert space. Let us introduce the new variables $u_{1}=\tilde{u}_{1}, u_{2}=\tilde{u}_{2}$, and $u_{3}=i \tilde{u}_{3}$, and let us define for $u=\left(u_{1}, u_{2}, u_{3}\right)$

$$
\begin{gathered}
\epsilon_{i j}^{\beta}(u)=\frac{1}{2}\left(\frac{\partial}{\partial x_{j}} u_{i}+\frac{\partial}{\partial x_{i}} u_{j}\right), 1 \leq i, j \leq 2, \\
\epsilon_{i 3}^{\beta}=\epsilon_{3 i}^{\beta}=\frac{1}{2}\left(\frac{\partial}{\partial x_{i}} u_{3}+\beta u_{i}\right),
\end{gathered}
$$

$1 \leq i \leq 3$

$$
\begin{gathered}
\epsilon_{33}^{\beta}=-\beta u_{3}, \\
\operatorname{div}^{\beta}(u)=\frac{\partial}{\partial x_{1}} u_{1}+\frac{\partial}{\partial x_{2}} u_{2}-\beta u_{3},
\end{gathered}
$$

and

$$
\sigma_{i j}^{\beta}=\lambda(x) \operatorname{div}^{\beta}(u) \delta_{i j}+2 \mu \epsilon_{i j}^{\beta}(u)
$$

$1 \leq i, j \leq 3$. 
We denote by $L_{n}^{2}$ the standard space of square integrable $c^{n}$ valued functions on $\mathbb{R}^{2}$ with scalar product

$$
(u, v)_{L_{n}^{2}}=\sum_{i=1}^{n} \int u_{i}(x) \bar{v}_{i}(x) d x .
$$

To use the standard notation we denote by $L^{2}=L_{1}^{2}$. For $m, n$ any positive integers we denote by $H_{m, n}$ the standard Sobolev space of all square integrable $\mathbb{C}^{n}$ valued functions defined on $\mathbb{R}^{2}$ all whose derivatives of order up to $m$ are square integrable functions with scalar product

$$
(u, v)_{H_{m, n}}=\sum_{|\alpha| \leq m}\left(D^{\alpha} u, D^{\alpha} v\right)_{L_{n}^{2}}
$$

where we use the standard multi-index notation for the derivatives

$$
D^{\alpha}=\frac{\partial^{\alpha_{1}}}{\partial x_{1}^{\alpha_{1}}} \frac{\partial^{\alpha_{2}}}{\partial x_{2}^{\alpha_{2}}} \quad, \quad|\alpha|=\alpha_{1}+\alpha_{2} .
$$

When $n=1$ we use the notation $H_{m}$ by simplicity.

Let us define the following operator $A(\beta)$ in $L_{3}^{2}$

$$
(A(\beta) u)_{i}=-\frac{1}{\rho}\left\{\sum_{j=1}^{2} \frac{\partial}{\partial x_{j}} \sigma_{i j}^{\beta}(u)-\beta \sigma_{i 3}^{\beta}(u)\right\}
$$

for $i=1,2, \quad$ and

$$
\begin{gathered}
(A(\beta) u)_{3}=-\frac{1}{\rho}\left\{\sum_{j=1}^{2} \frac{\partial}{\partial x_{j}} \sigma_{3 j}^{\beta}(u)+\beta \sigma_{33}^{\beta}(u)\right\}, \\
-4-
\end{gathered}
$$




$$
D(A(\beta))=\left\{u \in H_{1,3}: A(\beta) u \in L_{3}^{2}\right\}
$$

As we prove below $A(\beta)$ is selfadjoint in $L_{3}^{2}$ and its essential spectrum is given by (this was proven before by [BDJ] in the case when also $\rho$ and $\mu$ are variable but with $\rho(x), \mu(x)$, and $\lambda(x)$ constant outside of a bounded set):

$$
\sigma_{\ell}(A(\beta))=\left[\frac{\mu}{\rho} \beta^{2}, \infty\right)
$$

For definitions concerning the spectrum see $[\mathrm{K}]$.

It follows from an elementary calculation that there is a solution to (1.1) of the form (1.8), (1.9) if and only if

$$
A(\beta) u=\omega^{2} u
$$

with $u \in D(A(\beta))$. Then (see [BDJ]) the problem of finding guided waves for (1.1) is formulated as the problem of eigenvalues and eigenvectors for the selfadjoint operator $A(\beta)$ is $L_{3}^{2}$.

In [BDJ] a detailed study has been carried out of the guided waves with $\omega^{2}<$ $\frac{\mu}{\rho} \beta^{2}$. In physical terms they corresponds to guided waves whose phase speed $\omega / \beta$ is smaller than the speed of the $S$ (shear waves) $V_{S}=\left(\frac{\mu}{\rho}\right)^{\frac{1}{2}}$. In mathematical terms they correspond to the study of the discrete spectrum of the operator $A(\beta)$. For related results in acoustic and electromagnetic waves see [Wi 1], [G], [We 1], [We 2], [B], and [B B]. 
The question that we address in this paper has the following physical interpretation: does there exist guided waves whose phase speed is greater than $V_{S}$. From the mathematical point of view the same question is formulated as follows: does there exist eigenvalues of the operator $A(\beta)$ which are embedded in the essential spectrum.

This is quite a subtle question. This problem was already addressed in [BDJ] but the only results obtained in this direction concern the properties of possible eigenfunctions associated with such eigenvalues and also partial non existence results. Namely if the functions $\rho(x), \mu(x)$, and $\lambda(x)$ are piecewise constant $A(\beta)$ has no eigenvalues in the interval $\left(\frac{\lambda_{\infty}+2 \mu_{\infty}}{\rho_{\infty}}, \infty\right)$ where $\rho_{\infty}, \mu_{\infty}$, and $\lambda_{\infty}$, are the values taken by $\rho(x), \mu(x)$, and $\lambda(x)$ in the complement of a bounded set.

For related results in the absence of eigenvalues embedded in the essential spectrum of the acoustic propagator in perturbed stratified media see [We 2] and [We 3].

In this paper we construct guided waves that correspond to eigenvalues, $\omega^{2}$, of $A(\beta)$ with $\frac{\mu}{\rho} \beta^{2}<\omega^{2}<\frac{\lambda_{\infty}+2 \mu}{\rho} \beta^{2}$. These guided waves have phase speed bigger than the one of shear waves but smaller than the phase speed of pressure waves at infinity, namely $V_{P}=\left(\frac{\lambda_{\infty}+2 \mu}{\rho}\right)^{\frac{1}{2}}$.

We first introduce some useful notations.

$$
\not_{S}=\left\{u \in L_{3}^{2}: \operatorname{div}^{\beta}(u)=0\right\} .
$$

$H_{S}$ is a closed subspace of $L_{3}^{2}$. Let us denote by $H_{P}=H_{S} \frac{1}{S}$ the orthogonal complement of $H_{S}$ in $L_{3}^{2}$. Then 


$$
L_{3}^{2}=\mathfrak{H}_{S} \oplus \mathfrak{H}_{P}
$$

Suppose that $u \in \mathcal{H}_{P}$. Then

$$
(u, v)_{L_{3}^{2}}=0
$$

$\forall v \in \mathcal{H}_{S}$. In particular take $v=\left(v_{1}, 0, \frac{1}{\beta} \frac{\partial}{\partial x_{1}} v_{1}\right), \quad v_{1} \in H_{1}$.

Then

$$
(u, v)=\left(u_{1}, v_{1}\right)+\left(u_{3}, \frac{1}{\beta} \frac{\partial}{\partial x_{1}} v_{1}\right)=0 .
$$

Then $u_{1}=\frac{1}{\beta} \frac{\partial}{\partial x_{1}} u_{3}$. Taking $v=\left(0, v_{2}, \frac{1}{\beta} \frac{\partial}{\partial x_{2}} v_{2}\right)$ with $v_{2} \in H_{1}$, we prove that $u_{2}=\frac{1}{\beta} \frac{\partial}{\partial x_{2}} u_{3}$.

Then

$$
\mathcal{H}_{P}=\left\{u \in L_{3}^{2}: u_{1}=\frac{1}{\beta} \frac{\partial}{\partial x_{1}} u_{3}, u_{2}=\frac{1}{\beta} \frac{\partial}{\partial x_{2}} u_{3}, u_{3} \in H_{1}\right\}
$$

Suppose that $u \in D(A(\beta)) \cap \mathcal{H}_{S}$. Then it follows from an elementary calculation that

$$
\operatorname{div}^{\beta}(A(\beta) u)=0
$$

Then $A(B)$ sends $D(A(\beta)) \cap \mathcal{H}_{S}$ into $\mathcal{H}_{S}$. It follows from an elementary computation that $A(\beta)$ is symmetric. Then also $A(\beta)$ sends $D(A(\beta)) \cap \mathcal{H}_{P}$ into $\mathfrak{H}_{P}$. 
It follows that $A(\beta)$ decomposes along the decomposition (1.23) of $L_{\mathfrak{9}}^{2}$.

$$
A(\beta)=A_{S}(\beta) \oplus A_{P}(\beta)
$$

It is mainly for this purpose that we have assumed that $\mu$ and $\rho$ are constant. where $A_{S}(\beta)$ and $A_{P}(\beta)$ are respectively the restrictions of $A(\beta)$ to $K_{S}$ and $k_{P}$.

Note that for $u \in D\left(A_{S}(\beta)\right)$

$$
A_{S}(\beta) u=\frac{\mu}{\rho}\left(-\Delta+\beta^{2}\right) u
$$

Let us denote by $\lambda_{S}$ the Hilbert space of all functions on $L_{2}^{2}$ such that $\sum_{i=1}^{2} \frac{\partial}{\partial x_{i}} u_{i} \in L^{2}$ with scalar product

$$
(u, v)_{\hat{R}_{S}}=(u, v)_{L_{2}^{2}}+\frac{1}{\beta^{2}}\left(\sum_{i=1}^{2} \frac{\partial}{\partial x_{i}} u_{i}, \sum_{i=1}^{2} \frac{\partial}{\partial x_{i}} v_{i}\right)_{L^{2}}
$$

Let $U_{S}$ be the following unitary operator from $\hat{k}_{S}$ onto $k_{S}$

$$
U_{S}\left(\begin{array}{l}
u_{1} \\
u_{2}
\end{array}\right)=\left(\begin{array}{l}
u_{1} \\
u_{2} \\
\frac{1}{\beta} \sum_{i=1}^{2} \frac{\partial}{\partial x_{i}} u_{i}
\end{array}\right)
$$

We define the operator $\hat{A}_{S}(\beta)$ as

$$
D\left(\hat{A}_{S}(\beta)\right)=\left\{u \in \hat{\mathcal{H}}_{S}: \Delta u \in \hat{H}_{S}\right\},
$$




$$
\hat{A}_{S}(\beta) u=\frac{\mu}{\rho}\left(-\Delta+\beta^{2}\right) u
$$

One easily checks by Fourier transform that $\hat{A}_{S}(\beta)$ is selfadjoint, that its spectrum coincides with the continuous spectrum and it is given by

$$
\sigma\left(\hat{A}_{S}(\beta)\right)=\sigma_{c}\left(\hat{A}_{S}(\beta)\right)=\left[\frac{\mu}{\rho} \beta^{2}, \infty\right)
$$

By an elementary calculation using (1.30) we obtain that

$$
A_{S}(\beta)=U \hat{A}_{S}(\beta) U^{-1}
$$

Let us denote by $\hat{H}_{P}$ the space $H_{1}$ with scalar product

$$
(u, v)_{\ell_{P}}=(u, v)_{L^{2}}+\frac{1}{\beta^{2}} \sum_{i=1}^{2}\left(\frac{\partial}{\partial x_{i}} u, \frac{\partial}{\partial x_{i}} v\right)_{L^{2}}
$$

Let $U_{P}$ be the unitary operator from $\hat{H}_{P}$ onto $\varkappa_{P}$ given by

$$
U_{P} u=\left[\begin{array}{c}
\frac{1}{\beta} \frac{\partial}{\partial x_{1}} u \\
\frac{1}{\beta} \frac{\partial}{\partial x_{2}} u \\
u
\end{array}\right]
$$

It follows from an elementary calculation using (1.27) that for $u \in D\left(A_{P}(\beta)\right)$

$$
\begin{aligned}
\left(A_{P}(\beta) u\right)_{i}= & \frac{\partial}{\partial x_{i}} c^{2}(x)\left(-\Delta+\beta^{2}\right) \frac{u_{3}}{\beta} \\
& -9-
\end{aligned}
$$


$i=1,2$,

$$
\left(A_{P}(\beta) u\right)_{3}=c^{2}(x)\left(-\Delta+\beta^{2}\right) u_{3}
$$

where

$$
c(x)=\left(\frac{\lambda(x)+2 \mu}{\rho}\right)^{\frac{1}{2}} .
$$

Denote

$$
\hat{A}_{P}(\beta)=U_{P}^{-1} A_{P}(\beta) U_{P}
$$

Using (1.38), and (1.42) we obtain that

$$
D\left(\hat{A}_{P}(\beta)\right)=\left\{u \in \hat{\mathscr{H}}_{P}: c^{2}(x)\left(-\Delta+\beta^{2}\right) u \in \hat{\mathfrak{K}}_{P}\right\}
$$

and

$$
\hat{A}_{P}(\beta) u=c^{2}(x)\left(-\Delta+\beta^{2}\right) u
$$

for $u \in D\left(\hat{A}_{P}(\beta)\right)$.

Note that for $u, v \in D\left(\hat{A}_{P}(\beta)\right)$

$$
\begin{gathered}
\left(\hat{A}_{P}(\beta) u, v\right)_{\hat{\lambda}_{P}}=\left(-c^{2} \Delta u, v\right)_{L^{2}}+\left(u,-c^{2} \Delta v\right)_{L^{2}}+ \\
-10-
\end{gathered}
$$




$$
+\frac{1}{\beta^{2}}\left(c^{2} \Delta u, \Delta v\right)_{L^{2}}+\beta^{2}\left(c^{2} u, v\right)_{L^{2}}
$$

Denote for $u, v \in H_{2}$

$$
\begin{gathered}
a(\beta)(u, v)=\left(-c^{2} \Delta u, v\right)_{L^{2}}+\left(u,-c^{2} \Delta v\right)_{L^{2}}+ \\
+\frac{1}{\beta^{2}}\left(c^{2} \Delta u, \Delta v\right)_{L^{2}}+\beta^{2}\left(c^{2} u, v\right)_{L^{2}}
\end{gathered}
$$

Note that

$$
\overline{a(\beta)(u, v)}=a(\beta)(v, u)
$$

\section{Moreover}

$$
\begin{aligned}
& a(\beta)(u, u)=\int c^{2}(x)\left|\frac{1}{\beta} \Delta u-\beta u\right|^{2} d x \geq \\
& \geq \int c_{-}^{2}\left|\frac{1}{\beta} \Delta u-\beta u\right|^{2} d x=c_{-}^{2}\left[\frac{1}{\beta^{2}}(\Delta u, \Delta u)_{L^{2}}\right. \\
& \left.+2 \sum_{i=1}^{2}\left(\frac{\partial}{\partial x_{i}} u, \frac{\partial}{\partial x_{i}} u\right)_{L^{2}}+\beta^{2}(u, u)_{L_{2}}\right] \geq c_{-}^{2} \beta^{2}(u, u)_{\hat{H}_{P}},
\end{aligned}
$$

where $c_{-}=\left(\frac{\lambda_{-}+2 \mu}{\rho}\right)^{\frac{1}{2}}$.

Then $a(\beta)(\cdot, \cdot)$ is a closed symmetric and bounded below quadratic form. By (1.45) its associated selfadjoint operator (see $[K])$ is $\hat{A}_{P}(\beta)$. By (1.48).

$$
\hat{A}_{P}(\beta)>c_{-}^{2} \beta^{2}
$$


Let us denote by $\hat{A}_{P, \infty}(\beta)$ the operator defined as in (1.43), (1.44) but with $c(x)$ replaced by $c_{\infty}=\left(\frac{\lambda_{\infty}+2 \mu}{\rho}\right)^{\frac{1}{2}}$.

By a simple argument using the Fourier transform we prove that $\hat{A}_{P, \infty}(\beta)$ has no eigenvalues and that its spectrum coincides with the essential spectrum and it is given by

$$
\sigma\left(\hat{A}_{P, \infty}(\beta)\right)=\sigma_{e}\left(\hat{A}_{P, \infty}(\beta)\right)=\left[c_{\infty}^{2} \beta^{2}, \infty\right)
$$

Let us denote by $K$ the Hilbert space consisting of $L^{2}$ with scalar product given by

$$
(u, v)_{\mathcal{K}}=\int c^{-2}(x) u(x) \bar{v}(x) d x
$$

Let us denote by $B(\beta)$ the selfadjoint operator in $\mathcal{k}$ defined as follows

$$
\begin{gathered}
D(B(\beta))=H_{2}, \\
B(\beta) u=c^{2}(x)\left(-\Delta+\beta^{2}\right) u,
\end{gathered}
$$

for $u \in D(B(\beta))$.

Let $B_{\infty}(\beta)$ be the selfadjoint operator in $L^{2}$ defined as in (1.52), (1.53) with $c_{\infty}$ instead of $c(x)$. One easily checks by Fourier transform that $B_{\infty}(\beta)$ is absolutely continuous with spectrum given by 


$$
\sigma\left(B_{\infty}(\beta)\right)=\left\{c_{\infty}^{2} \beta^{2}, \infty\right)
$$

Since the norms of $\mathcal{H}$, and $L^{2}$ are equivalent we can consider $B(\beta)$ as a operator in $L^{2}$ with domain $H_{2}$, and $(B(\beta))^{-1}$ as a bounded operator from $L^{2}$ into $H_{2}$. Then in $L^{2}$

$$
\begin{gathered}
(B(\beta))^{-1}-\left(B_{\infty}(\beta)\right)^{-1}=\frac{1}{c_{\infty}^{2}}\left(-\Delta+\beta^{2}\right)^{-\frac{1}{2}}\left(\left(c_{\infty}^{2}-c^{2}\right)\left(-\Delta+\beta^{2}\right)^{-\frac{1}{2}}\right)^{*} \\
\left(-\Delta+\beta^{2}\right)(B(\beta))^{-1}
\end{gathered}
$$

By local Rellich compactness and (1.7) (see [Wi 2]) $\left(c_{\infty}^{2}-c^{2}\right)\left(-\Delta+\beta^{2}\right)^{-\frac{1}{2}}$ is a compact operator on $L^{2}$. It follows (see [S]) that $B(\beta)$ and $B_{\infty}(\beta)$ have the same essential spectrum (note that the spectrum of $B(\beta)$ as a operator in $k$ and in $L^{2}$ is the same). Then the essential spectrum of $B(\beta)$ is given by

$$
\sigma_{e}(B(\beta))=\left[c_{\infty}^{2} \beta^{2}, \infty\right)
$$

Note that $\hat{H}_{P} \subset \mathcal{K}$. We denote by

$$
\left.\left((B(\beta))^{-1}-\left(B_{\infty}(\beta)\right)^{-1}\right)\right|_{\hat{H}_{P}},
$$

the restriction of $(B(\beta))^{-1}-\left(B_{\infty}(\beta)\right)^{-1}$ to $\hat{H}_{P}$, where to simplify the notation we avoid the use of identification operators. By (1.44) we have that 


$$
\left(\hat{A}_{P}(\beta)\right)^{-1}-\left(\hat{A}_{P, \infty}(\beta)\right)^{-1}=\left.\left((B(\beta))^{-1}-\left(B_{\infty}(\beta)\right)^{-1}\right)\right|_{\hat{R}_{P}} .
$$

since $(B(\beta))^{-1}-\left(B_{\infty}(\beta)\right)^{-1}$ is compact from $L^{2}$ into $H_{1}$ the right hand side of (1.58) is compact in $\hat{H}_{P}$ (note that the norms of $H_{1}$ and $\hat{\mathscr{H}}_{P}$ are equivalent) and it follows that $\hat{A}_{P}(\beta)$ and $\hat{A}_{P, \infty}(\beta)$ have the same essential spectrum. Then by (1.50)

$$
\sigma_{e}\left(\hat{A}_{P}(\beta)\right)=\left[c_{\infty}^{2} \beta^{2}, \infty\right)
$$

Let us summarize the results that we have obtained about $A(\beta) . A_{S}(\beta)$ and $A_{P}(\beta)$ are selfadjoint because they are respectively unitarily equivalent to $\hat{A}_{S}(\beta)$ and $\hat{A}_{P}(\beta)$. It follows from (1.29) that $A(\beta)$ is selfadjoint. By (1.35) and (1.49)

$$
A(\beta)>\frac{\mu}{\rho} \beta^{2}
$$

and moreover (see also (1.59)) the essential spectrum of $A(\beta)$ is given by

$$
\sigma_{e}(A(\beta))=\sigma_{e}\left(A_{S}(\beta)\right) \cup \sigma_{e}\left(A_{P}(\beta)\right)=\left[\frac{\mu}{\rho} \beta^{2}, \infty\right)
$$

Since $A_{S}(\beta)$ has no eigenvalues any eigenvector of $A(\beta)$ will have zero component along $\varkappa_{S}$ (see (1.24)) that is to say it will actually be an eigenvector of $A_{P}(\beta)$ in $k_{p}$

Suppose there is an eigenvalue $\omega^{2}>c_{\infty}^{2} \beta^{2}$ of $A(\beta)$. Then for some $u \in \mathscr{H}_{P}, u \neq 0$ 


$$
A_{P}(\beta) u=\omega^{2} u
$$

By (1.40)

$$
c^{2}(x)\left(-\Delta+\beta^{2}\right) u_{3}=\omega^{2} u_{3},
$$

and then

$$
\left(-\Delta+\omega^{2}\left(\frac{1}{c_{\infty}^{2}}-\frac{1}{c^{2}}\right)\right) u_{3}=\left(\frac{\omega^{2}}{c_{\infty}^{2}}-\beta^{2}\right) u_{3} .
$$

If we assume that in (1.7) $\delta>1$ it follows from a standard result in the absence of eigenvalues of Schrödinger operators (see $[\mathrm{EK}]$ ) that $u_{3}=0$, and by (1.27) $u=0$. Then in this case $A(\beta)$ has no eigenvalues $\omega^{2}>c_{\infty}^{2} \beta^{2}$.

What about eigenvalues with $\frac{\mu}{\rho} \beta^{2}<\omega^{2}<c_{\infty}^{2} \beta^{2}$. These eigenvalues will be embedded in the essential spectrum of $A(\beta)$. However since the associated eigenvector, $u$, will belong to $\varkappa_{P}$ it will actually be an eigenvector of $A_{P}(\beta)$ :

$$
A_{P}(\beta) u=\omega^{2} u
$$

and since $\omega^{2}<c_{\infty}^{2} \beta^{2}, \omega^{2}$ will be a discrete eigenvalue of $A_{P}(\beta)$. This makes the existence of guided waves with $c_{-}^{2} \beta^{2}<\omega^{2}<c_{\infty}^{2} \beta^{2}$ quite plausible and on spite of the fact that they are embedded in the essential spectrum of $A(\beta)$ they will be stable in the sense that they correspond to the discrete spectrum of $A_{P}(\beta)$. 
We now proceed to state our result on the existence of these guided waves.

\section{Theorem I}

The operator $A(\beta), \beta>0$, is selfadjoint in $L_{3}^{2},(1.60)$ is satisfied and it decomposes as in (1.29). If (1.7) is true its essential spectrum is given by (1.61).

The eigenyectors of $A(\beta)$ have zero component along $H_{S}$ (see (1.24)) and they are solution of the equation (1.62) with $\omega^{2}$ the corresponding eigenvalue. If in (1.7) $\delta>1, A(\beta)$ has no eigenvalues $\omega^{2}$, with $\omega^{2}>c_{\infty}^{2} \beta^{2}$, where $c_{\infty}=\left(\frac{\lambda_{\infty}+2 \mu}{\rho}\right)^{\frac{1}{2}}$.

$A(\beta)$ has an eigenvalue $\omega^{2}$ if and only if $\omega^{2}$ is an eigenvalue of $B(\beta)$ in $L^{2}$ (see $(1.52),(1.53))$

If $v(x)$ is an eigenvector of $B(\beta)$ the corresponding eigenvector $u(x)$ of $A(\beta)$ is given by

$$
\begin{gathered}
u_{1}=\frac{1}{\beta} \frac{\partial}{\partial x_{1}} v, \\
u_{2}=\frac{1}{\beta} \frac{\partial}{\partial x_{2}} v \\
u_{3}=v .
\end{gathered}
$$

Q.E.D. 


\section{Corollary II}

Suppose that (1.7) is satisfied and that $\lambda_{-}<\lambda_{\infty}$.

Then there is an increasing sequence of real numbers $\beta_{m}, m=1,2,3 \ldots$, such that when $\beta_{m}<\beta \leq \beta_{m+1}, A(\beta)$ has at least $m$ eigenvalues $\left\{\omega_{1}^{2}(\beta) \leq \omega_{2}^{2}(\beta) \ldots \leq\right.$ $\left.\omega_{m}^{2}(\beta)\right\}$, counted with their multiplicities, and such that

1) $\forall m \geq 1, \beta \rightarrow \omega_{m}(\beta)$ is locally Lipschitz continuous, $\beta \rightarrow \omega_{m}^{2}(\beta)$ is strictly increasing, $\beta \rightarrow \omega_{m}^{2}(\beta)-c_{-}^{2} \beta^{2}$ is increasing, and $\beta \rightarrow \omega_{m}^{2}-\beta^{2} c_{+}^{2}$ is decreasing, where $c_{+}=\left(\frac{\lambda_{+}+2 \mu}{\rho}\right)^{\frac{1}{2}}$.

2) $\forall \beta>\beta_{m}, \quad c_{-}^{2} \beta^{2}<\omega_{m}^{2}(\beta)<c_{\infty}^{2} \beta^{2}$,

3) $\lim _{\beta \rightarrow \infty} \frac{\omega_{m}^{2}(\beta)}{\beta^{2}}=c_{-}^{2}, \lim _{\beta \backslash \beta_{m}} \frac{\omega_{m}^{2}(\beta)}{\beta^{2}}=c_{\infty}^{2}$.

4) Let $\lambda_{1}(x), \lambda_{2}(x)$ be two measurable real valued functions satisfying (1.5) to (1.7.), let $A_{i}(\beta), \quad i=1,2$, be the corresponding operators defined as in (1.18), (1.19) with $\lambda_{i}(x)$, and let $\omega_{i, m}(\beta), \beta_{i, m}, i=1,2$, be as above. Then if $\lambda_{1}(x) \geq \lambda_{2}(x)$, p.p., we have that $\omega_{1, m}(\beta) \geq \omega_{2, m}(\beta), \forall \beta \geq \beta_{1, m} \geq \beta_{2, m}$

5) The eigenvalues $\omega_{m}(\beta)$ are continuous on $\lambda(x)$ in the following sense. Let $\lambda_{i}(x), i=1,2,3, \ldots$ be measurable real valued functions satisfying $(1.5),(1.6)$ and $(1.7)$ with fixed constants $C$ and $\delta$, and with $\delta>2$. Let $\omega_{i, m}(\beta), \beta_{i, m}$ be as above for the operator $A_{i}(\beta)$ with $\lambda_{i}(x), \quad i=1,2, \ldots$ Then if

$$
\begin{gathered}
\lim _{i \rightarrow \infty} \text { ess } \sup \left|\lambda_{i}(x)-\lambda(x)\right|=0 \\
-17-
\end{gathered}
$$


we have that if $\beta>\beta_{m}$, then for $i$ large enough $\beta>\beta_{i, m}$, and

$$
\lim _{i \rightarrow \infty} \omega_{i, m}(\beta)=\omega_{m}(\beta)
$$

where the limit is uniform for $\beta$ in compact sets of $\left(\beta_{m}, \infty\right)$.

Q.E.D.

We complete the proof of Theorem I in section II where we give the proof of Corollary II.

Corollary II follows from Theorem I once the corresponding results are proven for $B(\beta)$. To do so we follow the method of $[B]$ and $[B B]$ where the closely related problem of a Schrödinger operator with a fixed potential of compact support and a variable coupling constant is considered. Moreover further results in the continuity of $\omega_{m}(\beta)$ can be obtained following [B] for instance.

In Section III we consider the case when $\lambda(x)$ is spherically symmetric.

A related result, namely the existence of embedded eigenvalues for the Dirichlet laplacian in domains with infinite boundary has recently been considered in [Wit 1], and [Wit 2]. 


\section{The Remaining Proofs}

The proof of Theorem I: Since the other results have already been proved in the introduction it only remains to prove that $A(\beta)$ has an eigenvalue $\omega^{2}$ if and only if $\omega^{2}$ is an eigenvalue of $B(\beta)$ in $L^{2}$ and that the corresponding eigenfunctions are related as in (1.66), (1.67), and (1.68).

Suppose that $\omega^{2}$ is an eigenvalue of $A(\beta)$ with eigenvector $u=\left(u_{1}, u_{2}, u_{9}\right)$. Then by (1.40)

$$
c^{2}(x)\left(-\Delta+\beta^{2}\right) u_{3}=\omega^{2} u_{3}
$$

It follows that $u_{3} \in H_{2}=D(B(\beta))$ and that $\omega^{2}$ is an eigenvalue of $B(\beta)$ with eigenvector $u_{3}$.

Suppose that $\omega^{2}$ is an eigenvalue of $B(\beta)$ with eigenvector $u_{3} \in H_{2}$. Then (2.1) is satisfied and it follows that $c^{2}(x)\left(-\Delta+\beta^{2}\right) u_{3} \in H_{2}$ since $\omega^{2}$ is a constant.

Define $u_{1}$ and $u_{2}$ as in (1.27).

It follows (see (1.39), (1.40)) that $u=\left(u_{1}, u_{2}, u_{3}\right) \varepsilon D\left(A_{P}(\beta)\right)$ and that

$$
A_{P}(\beta) u=\omega^{2} u
$$

Q.E.D

The proof of Corollary II: By Theorem I we only have to prove the statements for $B(\beta)$. To do so we follow the proof given in $[\mathrm{B}]$ and $[\mathrm{BB}]$ in the case of a two 
dimensional Schrödinger operator with a fixed potential of compact support, and a variable coupling constant. To make the paper self contained and for the reader's convenience we briefly indicate the main points of the proof.

Note that for $v \in D(B(\beta))$

$$
(B(\beta) v, v)_{\mathcal{X}}=\sum_{i=1}^{2}\left(\frac{\partial}{\partial x_{i}} v, \frac{\partial}{\partial x_{i}} v\right)_{L^{2}}+\beta^{2}(v, v)_{L^{2}} \geq c_{-}^{2} \beta^{2}(v, v)_{\mathcal{X}}
$$

where $c_{-}=\left(\frac{\lambda_{-}+2 \mu}{\rho}\right)^{\frac{1}{2}}$.

Then $B(\beta) \geq c_{-}^{2} \beta^{2}$. Moreover by (2.3) $B(\beta) v=c_{-}^{2} \beta^{2} v$ implies that $\frac{\partial}{\partial x_{i}} v=0$, and then $v=0$. It follows that

$$
B(\beta)>c_{-}^{2} \beta^{2},
$$

and that the discrete spectrum of $B(\beta)$ is contained on $\left(c_{-}^{2} \beta^{2}, c_{\infty}^{2} \beta^{2}\right)$ (recall that $\left.\sigma_{c}(B(\beta))=\left[c_{\infty}^{2} \beta^{2}, \infty\right)\right)$

Let us denote by $b(\beta)$ the quadratic form associated with $B(\beta)$. Then

$$
b(\beta)(u, v)=\sum_{i=1}^{2}\left(\frac{\partial}{\partial x_{i}} u, \frac{\partial}{\partial x_{i}} v\right)_{L^{2}}+\beta^{2}(u, v)_{L^{2}}
$$

for $u, v \in D(b(\beta))=H_{1}$.

By the Min-Max characterization of the eigenvalues below the essential spectrum (see [RS] Theorem XIII.I) we can define $\omega_{m}^{2}(\beta)$ as 


$$
\omega_{m}^{2}(\beta)=\sup _{v_{1} \ldots v_{m-1}} \inf _{u \in D(B(\beta)),\|u\|_{\mathcal{X}}=1, u \in\left[v_{1}, \ldots, v_{m-1}\right]^{\perp}} b(\beta)(u, u)
$$

where $\left[v_{1}, \ldots, v_{m-1}\right]^{\perp}$ denotes the orthogonal complement in $\forall$ to the subspace generated by $v_{1}, \ldots, v_{m-1} \cdot \omega_{m}^{2}(\beta)$ is an eigenvalue of $B(\beta)$ below the essential spectrum if and only if $\omega_{m}^{2}(\beta)<c_{\infty}^{2} \beta^{2}$. It follows from an elementary computation that for $\psi \in D(B(\beta)), \quad\|u\|_{\mathcal{X}}=1$, and $\beta^{\prime}>\beta$

$$
c_{-}^{2}\left(\beta^{\prime}+\beta\right) \leq \frac{b\left(\beta^{\prime}\right)(u, u)-b(\beta)(u, u)}{\beta^{\prime}-\beta} \leq c_{+}^{2}\left(\beta^{\prime}+\beta\right) .
$$

Then by (2.6)

$$
c_{-}^{2}\left(\beta^{\prime}+\beta\right) \leq \frac{\omega_{m}^{2}\left(\beta^{\prime}\right)-\omega_{m}^{2}(\beta)}{\beta^{\prime}-\beta} \leq c_{+}^{2}\left(\beta^{\prime}+\beta\right)
$$

By (2.8) the function $\beta \rightarrow \omega_{m}(\beta)$ is strictly increasing and locally Lipschitz continuous. Then $\omega_{m}^{2}(\beta)$ is differentiable p.p., and

$$
2 c_{-}^{2} \beta \leq \frac{d}{d \beta} \omega_{m}^{2}(\beta) \leq 2 c_{+}^{2} \beta .
$$

By (2.9) $\beta \rightarrow \omega_{m}^{2}-c_{-}^{2} \beta^{2}$ is increasing, and $\beta \rightarrow \omega_{m}^{2}(\beta)-c_{+}^{2} \beta^{2}$ is decreasing.

Let us now study the behavior of $\omega_{m}^{2}(\beta)$ when $\beta \rightarrow \infty$. In order to do that we use the following characterization of the $\omega_{m}^{2}(\beta)$ (see [DS] pages 1543, and 1544, and [B] Annexe in pages 277, to 285 where a proof is given). 


$$
\omega_{m}^{2}(\beta)=\inf _{V_{m} \in M_{m}(D(B(\beta)))} \sup _{\substack{v \in V_{m} \\ v \neq 0}} \frac{b(\beta)(v, v)}{(v, v)_{\mathcal{H}}},
$$

where $M_{m}(D(B(\beta)))$ is the family of all subspaces of $D(B(\beta))$ of dimension $m$.

We denote by $B_{R}$ the ball of center zero and radius $R$ in $\mathbb{R}^{2}$. Given $\epsilon>0$ take $R>0$ such that

$$
\int_{B_{R}}\left(\frac{1}{c_{-}^{2}}-\frac{1}{c^{2}(x)}\right) d x \leq \epsilon R^{2}
$$

and let $0<\lambda_{1} \leq \lambda_{2} \leq \ldots \leq \lambda_{m}$ be the first $m$ eigenvalues of $-\Delta$ with Dirichlet boundary condition on $\partial B_{\rho}$, with eigenvectors $v_{1}, v_{2}, \ldots, v_{m}$ normalized such that $\left\|v_{i}\right\|_{L^{2}}=c_{-, i} i=1,2, \ldots m$.

Denote by $V_{m}^{D}$ the subspace of $\mathcal{H}$ generated by $v_{1} \ldots, v_{m}$ (extended by zero to $\left.\mathbb{R}^{2} \backslash B_{R}\right)$. It follows from (2.10) and an elementary calculation that

$$
\begin{aligned}
& \omega_{m}^{2}(\beta)-c_{-}^{2} \beta^{2} \leq \sup _{\substack{v \in V_{m}^{D} \\
\|v\|_{L^{2}=c_{-}}}} \sum_{i=1}^{2}\left(\frac{\partial}{\partial x_{i}} v, \frac{\partial}{\partial x_{i}} v\right)_{L_{2}}+ \\
& +\beta^{2} c_{\infty}^{2} m \in R^{2} \sup _{1 \leq i \leq m}\left\|v_{i}\right\|_{\infty}^{2} .
\end{aligned}
$$

Where $\left\|v_{i}\right\|_{\infty}=e s s \sup \left|v_{i}(x)\right|$.

Denote by $\gamma_{i}, \quad i=1,2 \ldots, m$ the first $m$ eigenvalues of $-\Delta$ in $L^{2}\left(B_{1}\right)$ with Dirichlet boundary condition on $\partial B_{1}$, with associated eigenvectors $\psi_{1}(x), \ldots, \psi_{m}(x)$. Then by scaling $\lambda_{i}=\frac{1}{R^{2}} \gamma_{i}, \quad v_{i}(x)=\frac{1}{R} \psi_{i}\left(\frac{x}{R}\right)$. By (2.12) and Sobolev imbedding theorem 


$$
\omega_{m}^{2}(\beta)-c_{-}^{2} \beta^{2} \leq \frac{c_{-}^{2} \gamma_{m}}{R^{2}}+C_{m} \beta^{2} \epsilon
$$

for some constant $C_{m}$ independent of $\beta$ and $\epsilon$. It follows from (2.13) that

$$
\lim _{\beta \rightarrow \infty} \frac{\left(\omega_{m}^{2}(\beta)-c_{-}^{2} \beta^{2}\right)}{\beta^{2}}=0
$$

and since $c_{-}<c_{\infty}$, for $\beta$ large enough $\omega_{m}^{2}(\beta)<c_{\infty}^{2} \beta^{2}$. Define

$$
\beta_{m}=\inf \left\{\beta>0: \omega_{m}^{2}(\beta)<c_{\infty}^{2} \beta^{2}\right\}
$$

Note that since $B(\beta)>c_{-}^{2} \beta^{2}$, we have that $\omega_{m}^{2}(\beta)>c_{-}^{2} \beta^{2}$, for $\beta>\beta_{m}$. Since $\omega_{m}^{2}(\beta)$ is a continuous function of $\beta$ we must have that

$$
\lim _{\beta \downarrow \beta_{m}} \omega_{m}^{2}(\beta)=c_{\infty}^{2} \beta_{m}^{2}
$$

by the definition of $\beta_{m}$.

Let $\lambda_{1}(x), \lambda_{2}(x)$ be as in (4) of Corollary II. Let $\mathcal{H}_{i}$ be $L^{2}$ with scalar product as in (1.51) with $c_{i}(x)=\left(\frac{\lambda_{i}(x)+2 \mu}{\rho}\right)^{\frac{1}{2}}$, and let $B_{i}(\beta)$, and $b_{i}(\beta)$ be defined respectively as in (1.52), (1.53), and (2.5), for $i=1,2$.

Then for $v \in H_{1}$

$$
\begin{gathered}
\frac{b_{1}(\beta)(v, v)}{(v, v)_{\mathcal{X}_{1}}} \geq \frac{b_{2}(\beta)(v, v)}{(v, v)_{\mathcal{X}_{2}}} \\
-23-
\end{gathered}
$$


and it follows from $(2.10)$ that

$$
\begin{aligned}
& \omega_{m, 1}(\beta) \geq \omega_{m, 2}(\beta), \\
& \forall \beta>\beta_{1, m} \geq \beta_{2, m} .
\end{aligned}
$$

Finally let us prove (5). Let $K$ be a compact set contained on $\left(\beta_{m}, \infty\right)$. Then by $(1.71)$ and $(2.10)$ there is a $i_{0}$ such that for $i \geq i_{0}, \quad K \subset\left(\beta_{i, m}, \infty\right)$. Take $i \geq i_{0}$ and suppose that $\omega_{i, m}(\beta) \geq \omega_{m}(\beta)$. Since $\beta>\beta_{m}$ there exists $v_{1}, \ldots v_{m}$, with $\left(v_{i}, \in\right.$ $D(B(\beta))$

$$
\left(v_{i}, v_{j}\right)_{N}=\delta_{i, j}
$$

and

$$
B(\beta) v_{i}=\omega_{m}^{2} v_{i}
$$

Denote by $V_{m}$ the subspace generated by $v_{1}, v_{2} \ldots, v_{m}$. It follows from $(2.10)$ and a simple computation that

$$
\begin{gathered}
\omega_{i, m}^{2}(\beta) \leq \omega_{m}^{2}(\beta)+\frac{c_{i,+}^{2}}{c_{+}^{2}} \omega_{m}^{2}(\beta) \sup _{\substack{\varphi \in V_{m} \\
\|\varphi\|_{\mathcal{M}}=1}} \int|\varphi|^{2}\left|\frac{1}{c_{i}^{2}}-\frac{1}{c^{2}}\right|_{d x}^{2} \\
\leq \omega_{m}^{2}(\beta)+C\left(\left|c_{i, \infty}-c_{\infty}\right|+\int d x\left(\left|c_{i}(x)-c_{i, \infty}\right|+\left|c(x)-c_{\infty}\right|\right)\right), \\
-24-
\end{gathered}
$$


where we used Sobolev imbedding theorem, and the constant $C$ is uniform for $\beta \in K$. If $\omega_{i, m}(\beta)<\omega_{m}(\beta)$ we repeat the argument above with the roles of $\omega_{i, m}(\beta)$ and $\omega_{m}(\beta)$ exchanged and we obtain that

$$
\left|\omega_{i, m}^{2}(\beta)-\omega_{m}^{2}(\beta)\right| \leq C\left(\left|c_{i, \infty}-c_{\infty}\right|+\int d x\left(\left|c_{i}(x)-c_{i, \infty}\right|+\left|c(x)-c_{\infty}\right|\right)\right)
$$

Then by (1.71)

$$
\lim _{i \rightarrow \infty} \omega_{i, m}(\beta)=\omega_{m}(\beta)
$$

uniformly for $\beta \in K$

Q.E.D 


\section{Spherically Symmetric Case}

We consider now the case when $\lambda(x)$ is spherically symmetric and takes two values

$$
\lambda(x)= \begin{cases}\lambda_{\infty}, & \text { for }|x| \geq R, \\ \lambda_{-}, & \text {for }|x|<R,\end{cases}
$$

where $R, \lambda_{-}, \lambda_{\infty}$, are positive and $\lambda_{-}<\lambda_{\infty}$.

In this case the eigenvalues of $B(\beta)$ are of multiplicity two and the corresponding eigenfunctions are of the form (in polar coordinates $r>0, \quad 0 \leq \theta \leq 2 \pi$ ).

$$
u(r, \theta)=u_{n}(r)(A \cos n \theta+B \sin n \theta),
$$

where $(A, B) \in \mathbb{R}^{2}, n=0,1,2, \ldots$, and

$$
u_{n}(r)= \begin{cases}A_{\infty} K_{n}\left(k_{\infty} r\right), & \text { for } r>R, \\ A_{0} \quad J_{n}\left(k_{0} r\right), & \text { for } r<R,\end{cases}
$$

where $J_{n}\left(k_{0} r\right)$ is Bessel's function, and $K_{n}\left(k_{\infty} r\right)$ is the associated modified Bessels function that goes to zero at infinity.

\section{Moreover}

$$
k_{\infty}=\left(\beta^{2}-\frac{\omega^{2}}{c_{\infty}^{2}}\right)^{\frac{1}{2}}, \quad k_{0}=\left(\frac{\omega^{2}}{c_{-}^{2}}-\beta^{2}\right)^{\frac{1}{2}}
$$

where $\omega^{2}$ is the eigenvalue, $c_{\infty}=\left(\frac{\lambda_{\infty}+2 \mu}{\rho}\right)^{\frac{1}{2}}$, and $c_{-}=\left(\frac{\lambda_{-}+2 \mu}{\rho}\right)^{\frac{1}{2}}$. Since $\beta^{2} c_{-}^{2}<\omega^{2}<$ 
$\beta^{2} c_{\infty}^{2}, k_{\infty}$ and $k_{0}$ are both real. In order that $u(r, \theta)$ belongs to $D(B(\beta))$ both $u_{n}(r)$, and $\frac{d}{d r} u_{n}(r)$ have to be continuous at $r=R$. These conditions imply that

$$
\begin{gathered}
k_{0} \frac{J_{n}^{\prime}\left(k_{0} R\right)}{J_{n}\left(k_{0} R\right)}=k_{\infty} \frac{K_{n}^{\prime}\left(k_{\infty} R\right)}{K_{n}\left(k_{\infty} R\right)}, \\
\frac{A_{\infty}}{A_{0}}=\frac{J_{n}\left(k_{0} R\right)}{K_{n}\left(k_{\infty} R\right)}=\frac{k_{0} J_{n}^{\prime}\left(k_{0} R\right)}{k_{\infty} K_{n}^{\prime}\left(k_{\infty} R\right)} .
\end{gathered}
$$

Equation (3.5) gives the dispersion relations. It can be considered as an equation in which $\omega$ is the unknown and $\beta$ a parameter. Once (3.5) has been solved (3.6) gives $A_{\infty}$ as a function of $A_{0}$, or $A_{0}$ as a function of $A_{\infty}$.

For each angular wave number $n$ we denote by $\beta_{n, j}, j=1,2,3 \ldots$ the set of thresholds corresponding to that wave number.

For $\beta_{n, j}<\beta \leq \beta_{n, j+1}, \quad$ (3.5) has exactly $j$ solutions, $\omega_{n, i}(\beta), 1 \leq i \leq j$

The thresholds $\beta_{n, j}$ associated to the modes of the form (3.3) can be characterized as the solutions of the equation that one obtains by taking the limit of (3.5) when $\omega$ tends to $\beta c_{\infty}$. As $k_{\infty}$ tends to zero we use the fact that (see [GR] page 970 , formula (12), and [MOS] page 151)

$$
\lim _{x \rightarrow 0} x \frac{K_{n}^{\prime}(x)}{K_{n}(x)}=-n,
$$

to obtain the equation 


$$
\beta R\left(\frac{c_{\infty}^{2}}{c_{-}^{2}}-1\right)^{\frac{1}{2}} \frac{J_{n}^{\prime}\left(\beta R\left(\frac{c_{\infty}^{2}}{c_{-}^{2}}-1\right)^{\frac{1}{2}}\right)}{J_{n}\left(\beta R\left(\frac{c_{\infty}^{2}}{c_{-}^{2}}-1\right)^{\frac{1}{2}}\right)}=-n
$$

\section{Acknowledgements}

A preliminary form of Theorem I and Corollary II was proved by both authors in November of 1988 when $R$. Weder was visiting INRIA. These preliminary results where announced in $[\mathrm{J}]$.

R. Weder thanks Prof. G. Chavent, Dr. P. Joly, Dr. G. Cohen, and members of IDENT for their kind hospitality at INRIA. 


\section{$\underline{\text { References }}$}

[A] Achenbach J.D. Wave Propagation in Elastic Solids. North Holland, 1978.

[B] Bonnet A.S. Analyse Mathēmatique de la Propagation de Modes Guidés dans les Fibres Optiques. Rapport ENSTA No. 229 (1988).

[BB] Bamberger A., Bonnet A.S. Mathematical Analysis of the Guided Modes of an Optical Fiber. S.I.A.M. Journal in Mathematical Analysis 21, 6, 1487-1510 (1990).

[BDJ] Bamberger A., Dermenjian Y., Joly P. Mathematical Analysis of the Propagation of Elastic Guided Waves in Heterogeneous Media. Rapport I.N.R.I.A. No. 1013, 1989.

[DS] Dunford N., and Schwarz J.T. Linear Operators. Part II. Interscience Publishers. New York, London, 1963.

[EK] Eastham, M.S.P., and Kalf, H. Schrödinger Type Operators with Continuous Spectrum. Research Notes in Mathematics 65. Pitman, Boston, London, Melbourne, 1982.

[G] Guillot J.C. Completude des Modes T.E. et T.M. pour un guide d'Ondes Optiques Planaire. Rapport INRIA No. 385 (1985).

[GR] Gradshteyn I. S., and Ryzhik I. M. Tables of Integrals, Series, and Products. Academic Press, New York, San Francisco, London, 1965. 
[J] Joly P. Un Nouveau Résultat d'Existence d'Ondes Guidées en Milieu Elastique Hétérogéne. C.R. Acad. Sci. Paris 309, I, 793-796 (1989).

[K] Kato T. Perturbation Theory for Linear Operators. Springer Verlag Berlin, Heidelberg, New York, 1976.

[MOS] Magnus W. Oberhettinger F., and Soni R. P. Formulas and Theorems for the Special Functions of Mathematical Physics. Springer Verlag, Berlin, Heidelberg, New York, 1966.

[RS] Reed M., and Simon B. Methods of Modern Mathematical Physics Volume IV: Analysis of Operators. Academic Press, New York, San Francisco, London, 1978.

[S] Schechter M. Spectra of Partial Differential Operators. North Holland, Amsterdam, London, 1971.

[We 1] Weder R. Spectral and Scattering Theory in Deformed Optical Wave Guides.J. für die reine und angew. Mathematik $\underline{390}, 130-169$ (1988).

[We 2] Weder R. Spectral and Scattering Theory for Wave Propagation in Perturbed Stratified Media. Applied Mathematical Sciences. Vol 87. Springer Verlag. Berlin, Heidelberg, New York, 1991.

[We 3] Weder R. Absence of Eigenvalues of the Acoustic Propagator in Deformed Wave Guides. Rocky Mountains Journal of Mathematics 18, No. 2, 495-503 (1988). 
[Wi 1] Wilcox C.H. Sound Propagation In Stratified Media. Applied Mathematical Science. Vol. 50. Springer Verlag, Berlin, Heidelberg, New York, 1984.

[Wi 2] Wilcox C. Scattering Theory for the D'Alembert Equation in Exterior Domains. Lecture Notes in Mathematics 442. Springer Verlag, Berlin, Heidelberg, New York, 1975.

[Wit 1] Witsch K. J. Examples of Embedded Eigenvalues for the Dirichlet Laplacian in Perturbed Waveguides. Mathematical Methods in the Applied Sciences $\underline{12}$ 91-93 (1990).

[Wit 2] Witsch K. J. Examples of Embedded Eigenvalues for the Dirichlet Laplacian in Domains with Infinite Boundaries. Mathematical Methods in the Applied Sciences 12 177-182 (1990). 
Imprimé en France.

.l'Institut National de Recherche en Informatique et en Automatique 
ISSN $0249-6399$ 